\title{
OtO Matching System: A Multi-strategy Approach to Instance Matching
}

\author{
Evangelia Daskalaki and Dimitris Plexousakis \\ Foundation of Research and Technology Hellas, Institute of Computer Science, Crete, Greece \\ \{eva, dp\} aics. forth.gr
}

\begin{abstract}
In this paper we describe an ontology to ontology (OtO) matching system which implements a novel instance matching algorithm. The proposed multi-strategy matching system is domain independent and fully customizable at any level. It optimizes the instance matching process by leveraging (a) the rich semantic knowledge from the schema matching results, (b) the implicit knowledge of the domain expert by capturing the identification power of the properties and (c) the probability estimation of the result's validity, in order to accurately detect the ontology instances that represent the same real-world entity. Furthermore we evaluate the system with the ISLab Instance Matching Benchmark in the Ontology Alignment Evaluation Initiative 2009 campaign and report the results.
\end{abstract}

Keywords: Ontology, instance matching, record linkage, entity resolution.

\section{Introduction}

Ontologies, as the means to conceptualize domain knowledge, have become the enabler of the fulfillment of the semantic web vision. Their aim is to make data sharable in order to make them understood and processed by computers with minimum human interference.

Due to the fact that ontologies are heterogeneous and distributed, it is necessary to discover the alignment across ontologies but also the alignments across their instances. In other words, it is necessary to achieve semantic interoperability across ontologies. In [44] ontology alignment between two ontologies is described as a function which gives as output a relationship between the vocabularies of the ontologies.

Several frameworks for ontology integration have been proposed. Their research efforts are mainly focused on the integration at the ontology schema level. For those that consider integrating data at the ontology instance level only a few systems deal with this problem. At the same time, the demand for high-quality ontology instance matching is crucial in the context of identity recognition, ontology population and semantic integration.

We propose a new multi-strategy OtO matching system that focuses on the ontology instance matching, but also implements schema alignment. The OtO matching system is domain independent and fully customizable by the domain expert at any level. We argue that, unless a system is fully customizable, it can not claim to be 
domain independent. The proposed system implements several different ontology matching processes, which can be roughly categorized into the processes that include schema matching algorithms and those that include instance matching algorithms.

The schema matching process of the proposed system leverages the lexical, semantic and syntactic similarities of the entities of the schema. As far as the instance matching process is concerned, it does not only rely on lexical similarity measures and pair-wise instance comparison of the instance properties. The system optimizes the instance matching process by leveraging (a) the rich semantic knowledge we gain from the output mappings of the schema matching process, (b) the implicit knowledge of the domain expert by capturing the identification power of the properties and (c) the probability calculation of the result's truth, in order to accurately and efficiently detect the ontology instances that represent the same real-world entity.

We evaluated the OtO matching system with the ISLab Instance Matching Benchmark (IIMB) where we achieved the best results in recall and the second best results in precision among all the systems that took part in the benchmark, with $100 \%$ recall and $97 \%$ precision.

The rest of this paper is organized as follows: In section 2 we give a formal definition of ontology instance matching. Furthermore, we analyze the ontology instance matching requirements. In section 3 we review the related scientific work done within the past years, as well as the related ontology instance matching frameworks. In section 4 we give a detailed description of the proposed OtO matching system, by focusing on the new instance matching algorithm. In section 4 we deal with the experiments and their results. We discuss the outcomes returned by running the test cases from the OAEI 2009 IIMB, in comparison with the other instance matching frameworks that took part in this benchmark. Finally, in section 6, we summarize the strengths and the weaknesses of the OtO matching systems and outline further research work.

\section{Instance Matching}

In contrast to ontology schema matching, instance matching deals with the actual individuals, not with the alignment of class structures or entity types. Instance matching has to deal with deciding whether two entity descriptions refer to the same individual. Thus, it crucially depends on measuring the similarity between sets of annotated instances.

As stated in [1] the application of instance matching is crucial in identity recognition, ontology population and semantic integration. Identity recognition refers to the capability of detecting whether two different resource descriptions refer to the same real-world entity, namely an individual. Ontology population refers to the need of supporting experts in managing ontology changes through advanced, and possibly automated, techniques [2,3]. It can be described as a part of ontology evolution, where ontology is evolved by acquiring new semantic descriptions of data extracted from heterogeneous data sources. For ontology population, instance matching plays a crucial role in order to correctly perform the insertion activity and discover the relationship between new incoming instances and the set of instances already existing in the 
ontology. The matchings produced by instance matching are exploited for clustering instances that are recognized as referring to the same real-world entity.

Finally, due to the increasing popularity of Semantic Web technologies, a novel attention on semantic integration issues has risen. For semantic integration, advanced techniques for ontology instance matching are required to correctly combine data describing individuals in different sources and to improve the accuracy of the schema ontology alignment process. The notion behind this is that the more significant the overlap of common instances of two ontology concepts is the more related these concepts are.

In our work we focus on identity recognition. Identity recognition is formally described in [4] as the matching procedure of two instances that belong to the same ontology or different ontologies, where the result can either be $\{$ match $\}$ or $\{$ no match . If the result is \{no match $\}$ then this denotes the fact that the two instances do not refer to the same real object. On the other hand if the result is $\{$ match $\}$ then this denotes the fact that both instances refer to the same real object.

\section{Related Work}

The problem of instance matching has been a topic of research for many years. It is also known as the problem of record linkage [5,6], duplicate detection [7], entity resolution [8], merge/purge [9], and object reconciliation or identification [10]. Authors in [11] focus on the idea of establishing co-reference information and preserving it, in order to build large scale networks of knowledge. Data integration and methods are also discussed in [12] and [13].

While the majority of existing methods were developed for the task of matching database records, modern approaches mostly focus on graph-based data representations extended by additional schema information. This allows us to describe it within the well-established ontology matching framework [14].

The ontology instance matching problem is seen in the literature from two different perspectives. In the first perspective the instance matching results are the means to derive more accurate schema matching results [15-17]. The second perspective uses the outcomes of the ontology schema matching in order to improve the instance matching process [18]. The notion behind the latter perspective is that if two ontology concepts are semantically correlated then it is likely that their instances refer to the same individuals, but if the concepts are not related then their instances will also not be related to each other.

As far as the matching techniques are concerned, authors in [44] differentiate the instance matching systems into those that use linguistic-based approaches, using names and textual descriptions and those that use constraint-based approaches, using element constraints such as data types, domains and key characteristics.

Following the differentiation mentioned above DSSim [22], A-Flood [23], CODI [25], SERIMI [28], AgreementMaker [29] and Zhishi.links [30] are linguistic-based systems. On the other hand ASMOV [20], RiMOM [19], COMA++ [24], FBEM [21], H-Match2.0 [26], LN2R [31], ObjectCoref [27] and OtO system use constraint-based approaches. 
In particular, the DSSim instance mapping process compares all the instance properties by using multi core processors and splitting up the large ontologies into smaller fragments. A-Flood uses firstly the schema matching results as input for the instance matching. Then, the alignment of the types of the instances is checked. CODI is a probabilistic -logical alignment system. It implements an approach which utilizes object-properties to determine the instances for which the similarity should be computed. In this system, the schemata are assumed to be the same. SERIMI extracts the instance labels and searches for instances in a target dataset that may have a similar label. Then it filters among the instances found in the target dataset, those that actually refer to the same entity in the real world as the source instance. The instance matching algorithm in AgreementMaker consists of doing a lookup using the label of the instance, the type, and querying against an index which returns a reasonable number of candidate target instances. The target instances are then ranked and eventually the best one is selected. Zhishi.links begins with the string similarity calculation and then with the semantic similarity calculation. Match candidates are sorted by their similarity scores.

The RiMOM system, ASMOV, FBEM, H-Match2.0, ObjectCoref and the OtO matching system are constraint-based systems, because they differentiate the significance of the instance properties with the use of the property weights (keycharacteristics). The weights' calculation though, is done differently for every system. In ASMOV and RiMOM weights are fixed and depend on the occurrence of each instance property. ObjectCoref system's architecture follows a common self-training framework. For calculating the similarity between the instances it uses fixed weights proportional to the frequency of their property values. In H-Match2.0 and FBEM, weights are fixed and calculated by using a vocabulary of key properties. OtO system also uses a vocabulary of key properties, but property weights are not fixed. They can be customized from the domain expert. In COMA++ system constraints are compared. COMA++ distinguishes between the General constraints, the Numerical constraints, and the Pattern constraints. In the match process the constraint-based matcher determines the similarity of two elements by comparing their previously identified constraints. The content-based matcher determines the similarity of two elements by executing a pair-wise comparison of instance values using a similarity function. The result is a similarity matrix, with each dimension representing the instances of one element. Finally, the reference reconciliation system (LN2R) is a knowledge-based, unsupervised system, based on two methods, a logical one called L2R and a numerical one called N2R. The Logical method for Reference Reconciliation (L2R) is based on the translation in first order logic (Horn rules) of some of the schema semantics. In order to complement the partial results of L2R, a Numerical method for Reference Reconciliation (N2R) is created. It exploits the L2R results and allows computing similarity scores for each pair of references.

The advantage of the OtO system is that, apart from using the property weights to cluster the properties of high importance, moreover these weights can easily be customized from the domain expert, in order to achieve the best results. Another novel feature of the OtO system is that it does not filter the results only with the commonly used threshold, but it also uses the property weights to calculate the trust ability of the results truth. All these advantages are analyzed in the following sections. 


\section{The OtO Matching System}

The proposed multi-strategy OtO matching system is domain independent and fully customizable at any level. It implements several different ontology matching strategies, which can be roughly categorized into the schema matching and the instance matching strategies. Our contribution in the OtO matching system is novel instance matching algorithm. In this section, we provide a detailed presentation of the matching process. The matching process consists of two process phases a) the schema matching, and b) instance matching.

The first phase of the matching process is the schema matching process. Depending on the domain expert's selections, the OtO matching system either proceeds with the schema matching process or not. If the user does not select to run a schema matching algorithm, the system assumes that the source schema and the target schema are the same and creates a "mirror mapping", which is stored into the database. In order to create the mirror mapping the OtO system reads the source schema and creates a mirror of it as the target schema.

If the domain expert has selected to proceed with the schema matching, then the OtO system follows the algorithm that is described in [33], which followed the work done in [34]. In [33], after storing the ontologies into the database as a directed acyclic graph, the schema matching algorithm follows a hybrid algorithm for matching the schemata. It is called hybrid algorithm because, depending on the domain expert's selections, the algorithm runs sequentially a lexicographic matching process, a semantic matching process and syntactic matching process between the schemata. In the lexicographic matching process, each pair of concepts is compared by using character-based metrics, token-based metrics, phonetic-based metrics and the WordNet ${ }^{\circledR}$ Thesaurus. Then it returns a similarity matrix of all the corresponding concepts. In the sequel, the semantic matching process calculates the semantic similarity for each pair of concepts, based on attributes/properties that characterize and describe the two concepts. Finally, both lexical and semantic similarities are taken into account for the next step, which is syntactic similarity. For each pair of concepts, the descendants' similarity is examined in order to produce the total similarity measurement. The final result is produced by combining lexical, semantic and syntactic similarity.

The schema matching results are presented to the end user in the form of a table with the source element, the target element and the similarity of each match. The domain expert can either accept the matches, or reject them, by unchecking the "accept" checkbox of each match. Furthermore, the domain expert can alter the automatically predefined weights of significance of each property match. The usage of these weights is described in detail in the next sections.

\subsection{The Instance Matching Process}

The second phase of the matching process is the ontology instance matching. This is the new feature of the OtO matching system which completes the OtO matching process by allowing the comparison of the instances. Through this procedure, the OtO matching system measures the similarity between sets of annotated instances. The goal of this process is to determine instances that represent the same real particulars. 
The OtO matching system introduces a combination of methods and algorithms for better accuracy, efficiency and effectiveness. In particular the instance matching algorithm leverages (a) the rich semantic knowledge we gain from the output mappings of the schema matching process, (b) the implicit knowledge of domain expert capturing the identification power of the properties with the use of property-weights and (c) the 2-phase filtering method of the results, in order to accurately and efficiently detect the ontology instances that represent the same real-world entity.

As far as the first is concerned, the OtO matching instance algorithm uses the outputs of the schema matching process. As a result only the instances that belong to semantically related classes are included in the instance matching algorithm. The intuition behind this is that the schema mappings contain semantic knowledge which is important for the efficient instance matching. If for example the schema matching returns a mapping between the "person" entity from ontology A and the "sportsperson" entity from Ontology B, then it is wise just to compare the instances of type "person" from otology A, with the instances of type "sportsperson" from ontology B, rather than comparing all the instances from ontology A with all the instances from ontology B.

Furthermore, the OtO system uses the keyword rules (vocabularies) in order to automatically detect the significant properties and assign them high property weights. By capturing the identification power of the properties, the OtO system can reduce the unnecessary comparisons of the property instances, and only compare the properties of the instances that can determine the identity recognition. An important feature of the system is that the domain expert can see the property weights and alter or correct them depending on his/her implicit knowledge on the ontologies.

As far as the third point is concerned, the OtO system uses two different filtering methods to determine whether a match is considered as confident or not. The first is the threshold filter and the second is the key-weight flag filter. If the weighted similarity of an instance pair is below the threshold then it is not considered as a match, otherwise it can be considered as match depending on the second filter. The second filter, the key-weight flag filter, is the probability of an instance pair to be a true match. Through the use of the property weights, the OtO matching system can calculate the probability of an instance pair to be true match or not.

\subsection{Property Weights}

The OtO instance matching algorithm uses the property weights in order to capture the identification power of the properties. The property weight is a real number in the range of $[0.0,1.0]$. This number shows how indicative a property match is to determining whether two instances refer to the same real object or not. In other words, the property weight indicates the probability of a property match with the same value to be true particular match. This probability includes two kind of stochastic processes, as also described in [35]: the probability distribution of the property and the probability distribution of the knowledge of the property. The first refers to how many instances share the same property value in the real world and the second refers to how many instances share the same property in a specific knowledge base.

In order to have a better understanding of previously mentioned stochastic processes, let us see an example. Assumingly we are comparing two instances of the 
ontology class "country", in order to indentify if these two instances refer to the same real country or not. This "country" class has two properties: (a) the "countrycurrency" and (b) the "country-name". For both of the instances the value of the property "country-currency" is "EUR". Given that 17 countries have as official currency the EUR, the probability of these two countries to refer to the same real country would be $5.89 \%$ (i.e., 1 out of 17). If the domain expert knew that only 10 out of the 17 are inserted in our knowledge base then the probability would be $10 \%$ that these two countries refer to the same real country.

As far as the property "country-name" is concerned the distribution of this property and the distribution of the knowledge of the property is the same; each country has a unique name. So the probability of two countries to refer to the same real country if they share the same name is $100 \%$. Thus, the weight of the property "country-name" would be would be 1.0 and the weight of the property "country-currency" would be 0.1 .

The domain expert is expected to alter the automatically inserted weights of each property-match or insert new ones in the matching process, if she considers it as necessary. A first attempt to insert weights to the properties is done automatically from the OtO system, by using keyword rules. These keyword rules can easily be customized by the domain expert. The property matches that include the specific keywords are given automatically the associated weight amount. Some examples of the keywords are: "name 1.0", "homepage 1.0", "coordinates 1.0", "website 0.9", etc. The other property matches are given zero weight. The domain expert is asked to correct the weights and insert more or delete the unnecessary ones according to her implicit knowledge.

As described above, through the use of weights the system is able to determine the matches that are crucial for the outcome and separate them from the matches that are not important. This is done in the matching results table, after the schema matching process. If the domain expert selects the weight of $\mathrm{w}_{\mathrm{i}}=0$, this means that the matching is not important for the result and thus does not match the values of the instance properties. On the other hand if he/she selects $\mathrm{w}_{\mathrm{i}}=1.0$ this means that the similarity of the property match is of high importance and may indicate that the property values match the entire instance. Thus, the OtO matching system is able to reduce the number of comparisons conducted, without losing any important information.

\subsection{The Key Weight Flag}

The key weight flag is an important feature added into the instance matching algorithm in order to provide more accurate and trustworthy results. The key weight flag is a flag which allows instance matches with similarity above threshold to be added in the mapping result. In order to determine this, the key weight flag is turned into true (green flag to pass) only if the matched instances have at least one property match with weight above or equal 0.5 (wi $>=0.5$ ).

$$
\text { Count }\left(\left[w_{1}, w_{2}, w_{3}, \ldots, w_{p}\right]>=0.5\right)>0
$$

If the matched instances have similarity above the threshold but all their property annotation weights are less that 0.5 then the match is not considered as trustworthy and is not added in the instance matches result. 


\subsection{Instance Matching Algorithm}

The algorithm begins by loading the schema mappings. For each class mapping returned from the schema matching, the algorithm returns the source class instances and the target class instances.

For each of the instances of the source and the target mapping class, the algorithm returns the property annotations and their values. If there are no class mappings then the algorithm compares all the instances with each other. If the property annotations of the instances are among the property mappings, then the algorithm checks the property weights of the match. If the weight is over the low limit of 0.0 , then the algorithm checks the property values. If the property values are references to other instances then it indicates the correct instance values. Once the values have been indicated the algorithm proceeds with the value matching algorithms.

The domain expert can select the string matching algorithms that will be applied on the instance values. There are many available instance matching algorithms. They contain character based metrics, token-based metrics, phonetic similarity metrics and semantic-based metrics. After applying the selected string matching algorithms, a weighted average of the property similarities is calculated. This process is followed for all the matching property annotations of each instance matching pair and the property similarities are stored in a property similarity matrix.

When there are no more properties to compare, a weighted average of the instance similarity is calculated this time for the instance pair with formula (1). The similarities $\operatorname{sim}=\left\{\operatorname{sim}_{1}, \operatorname{sim}_{2}, \operatorname{sim}_{3}, \operatorname{sim}_{\mathrm{p}}\right\}$ represent the similarities of each property pair and the weights $\mathrm{w}=\left\{\mathrm{w}_{1}, \mathrm{w}_{2}, \mathrm{w}_{3}, \ldots, \mathrm{w}_{\mathrm{p}}\right\}$ represent the weights of each matching pair which are given by the domain expert.

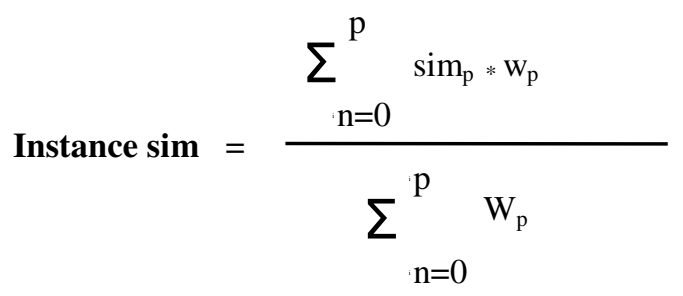

If the calculated instance similarity is above the given threshold then the instance pair is an accurate match and might be added in the matching results. If it will be added in the matching result or not, depends on the "key-weight flag".

Unlike the schema matching algorithm, in the instance matching algorithm every source instance is allowed to match with no more than one target instance (1:1). That of course is based on the fact, that the aim of the instance matching algorithm is the identity recognition, so the system returns only the best target instance match for a given source instance. Figure 1 shows the instance matching algorithm in pseudocode. 


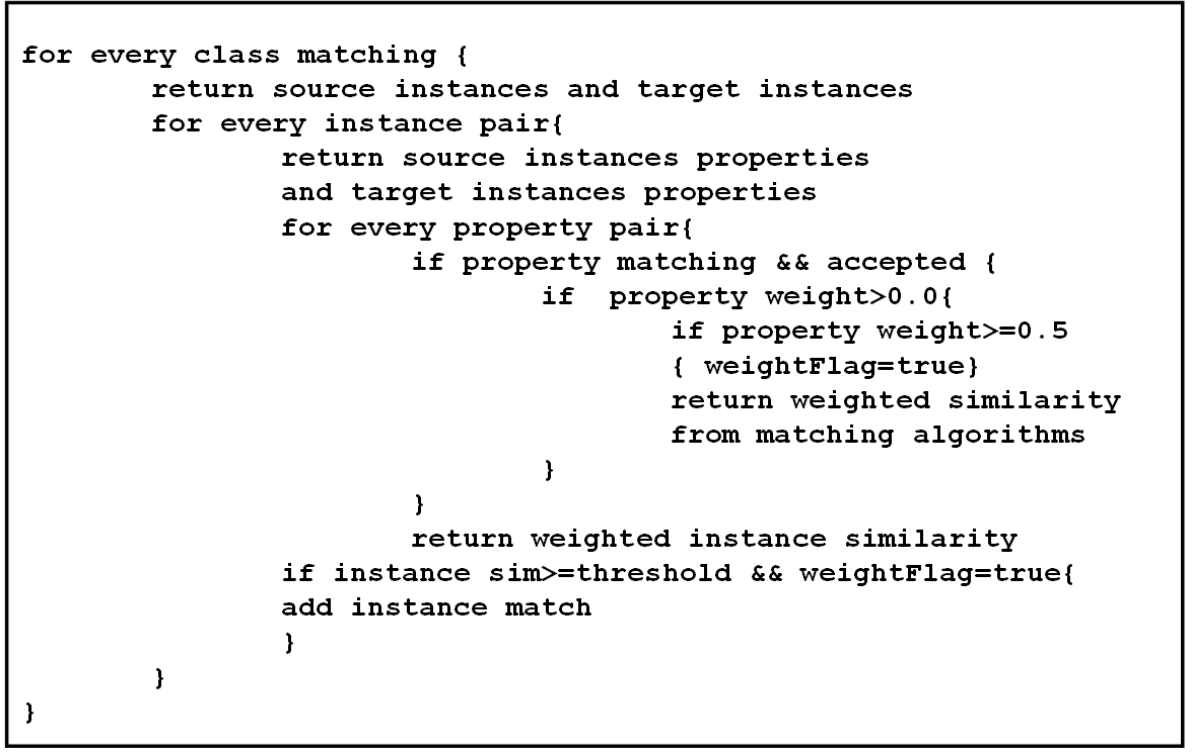

Fig. 1. Instance matching algorithm in pseudo-code

\section{Experiments and Results}

In order to evaluate the matching system and test the instance matching algorithm we have used the ISLab Instance Matching Benchmark (IIMB) [36]. The IIMB is a benchmark that is generated automatically, starting from one data source that is automatically modified according to various criteria. The original data source contains OWL/RDF data about actors, sports, persons, and business firms provided by the OKKAM European project [37].

The benchmark is composed by 37 test cases. For each test case it is required to match the original data source (source ontology) against a new data source (target ontology). The original data source contains 222 different instances and the new data sources contain from 222 to 1700 different instances. Each test case contains a modified ABox (abox.owl + tbox.owl) and the corresponding mapping with the instances in the original ABox (Golden standard).

The IIMB benchmark was chosen because it contains many test sets which introduced different kinds of challenges as listed below:

- Test case 01: Contains an identical copy of the original data source (instance IDs are randomly changed)

- Test case 02 - Test case 10: Value transformations (i.e., typographical errors simulation, use of different standards for representing the same information). In order to simulate typographical errors, property values of each instance are randomly modified. Modifications are applied on different subsets of the instances property 
values, with varying levels of difficulty (i.e., introducing a different number of errors).

- Test case 11 - Test case 19: Structural transformations (i.e., deletion of one or more values, transformation of datatype properties into object properties, separation of a single property into more properties).

- Test case 20 - Test case 29: Logical transformations (i.e., instantiation of identical individuals into different subclasses of the same class, instantiation of identical individuals into disjoint classes, instantiation of identical individuals into different classes of an explicitly declared class hierarchy).

- Test case 030 - Test case 037: Several combinations of the previous transformations.

Furthermore the IIMB Benchmark was firstly introduced in the Ontology Alignment Evaluation Initiative 2009 campaign [38]. Thus, apart from having a Golden Standard to compare our results we also have other systems' results to be compared against. The other systems that participated in the IIMB benchmark 2009 campaign are the Aflood system [39], the ASMOV system [40], the DSSim system [41], the HMatch system, the FBEM system [42] and the RiMOM system [43].

\subsection{Overall Conclusions of the Test Cases}

The results of the Harmonic mean of the test cases, as reported in [45], have shown that the OtO system is ranked in the first position in terms of recall among all systems with recall $=100 \%$ and the second position in terms of precision with precision $=97 \%$ as shown in Table 1.

Table 1. Harmonic mean of test cases

\begin{tabular}{|l|l|l|l|}
\hline & Prec. & Rec. & FMeas. \\
\hline AFlood & 0.92 & 0.87 & 0.89 \\
\hline ASMOV & 1.00 & 0.98 & 0.99 \\
\hline DSSim & 0.92 & 0.48 & 0.63 \\
\hline HMatch 2.0 & 0.89 & 0.93 & 0.91 \\
\hline FBEM & 0.16 & 0.75 & 0.27 \\
\hline RiMOM & 0.96 & 0.98 & 0.97 \\
\hline OtO & 0.97 & 1.00 & 0.98 \\
\hline
\end{tabular}

Many of the test cases contained skewed data with typographical errors. An example of test case 10 can be seen in Figure 2. This example shows two instances from the source ontology and the target ontology respectively, that are reported to be a correct match according to the Golden Standard. The OtO matching system identified that the significant instance properties that should be compared are the "cogito-name" and the "cogito-first-sentence". By applying character based metrics, token-based metrics and phonetic similarity metrics, the OtO matching system correctly indicated that these two instance where a true match. 


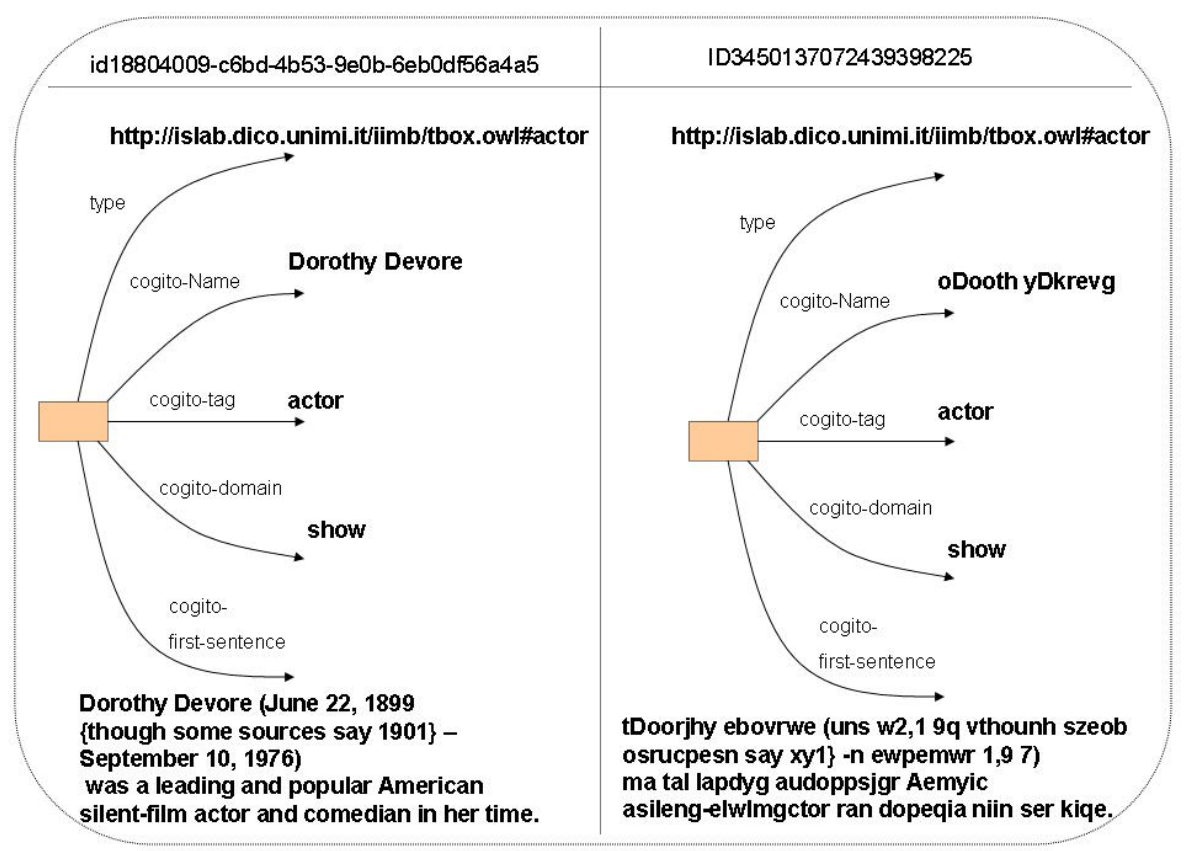

Fig. 2. Example of instances snapshot from test case 10

In some other test cases random properties have been removed, where some of these have been critical for the identification of the instances. Due to the 2-phase filtering method, the OtO matching system returned only those results where the confidence level of the results truth was high. Thus, when the instance properties were not significant enough, then the key weight flag prevented these results from being added in the OtO matching instances list. Figure 3 shows an example of test case 31.

Figure 3 shows two instances from the source ontology and the target ontology respectively. The Golden Standard reported that these to instances are a true match, but the OtO matching system did not return them as a correct match. The reason is that the key-weight flag filter indicated that the properties "cogito-domain" and "type" were not significant enough (the property weights were below 0.5 ) to determine if these two instances refer to the same real object.

Last but not least, the OtO matching system reported F-measure $=1$ in $84 \%$ of the test cases. This means that in eight out of ten cases the system returned all the correct answers and no false answer. What's more, another success of the OtO system is that it was ranked in the first position in F-measure in 32out of 37 test cases. For the results of all the test cases, please refer to: http://www.ics.forth.gr/ eva/ oto_Matching_System_Results.pdf 


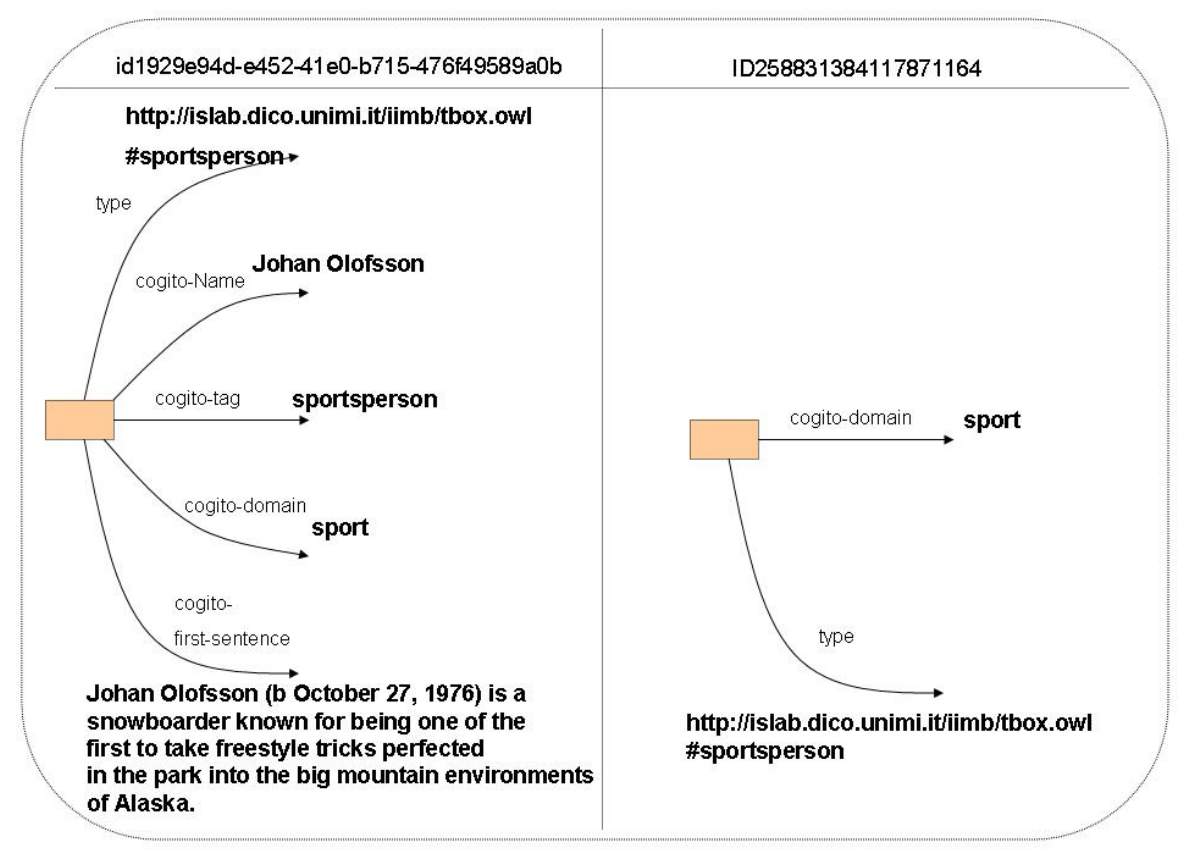

Fig. 3. Example of instances snapshot from test case 31

\section{Conclusions and Future Work}

In this paper, we presented the OtO matching system, which is a multi-strategy ontology to ontology matching system. It was shown that it is a domain independent and fully customizable matching system at any level, i.e. schema level or instance level.

In order to evaluate the matching system and test the instance matching algorithm we have used the ISLab Instance Matching Benchmark (IIMB) from the OAEI 2009 campaign. The IIMB is a benchmark that is generated automatically, starting from one data source that is automatically modified according to various criteria. The benchmark results have shown that the OtO matching system has returned one of the best results. In particular, it returned the best results in recall and the second best results in precision, among seven systems.

The success of the system is grounded on the carefully designed and analyzed instance matching process. Specifically speaking, the success is due to the leverage of (a) the rich semantic knowledge we gain from the output mappings of the schema matching process, (b) the implicit knowledge of the domain expert by capturing the identification power of the properties and (c) the probability calculation of the result's truth, in order to accurately and efficiently detect the ontology instances that represent the same real-world entity. 
We aim to work on the OtO matching system in order to make it even more efficient when it comes to large ontologies with millions of instances. Our focus will be on the use of multi core processors and the splitting large ontologies into smaller.

Acknowledgement. This work has been supported by the eHealthMonitor project (http://www.ehealthmonitor.eu) and has been partly funded by the European Commission under contract FP7-287509.

\section{References}

1. Castano, S., Ferrara, A., Montanelli, S., Lorusso, D.: Instance Matching for Ontology Population. In: Proceedings of the Sixteenth Italian Symposium on Advanced Database Systems, SEBD 2008, pp. 22-25 (2008)

2. Kondylakis, H., Plexousakis, D.: Exelixis: evolving ontology-based data integration system. In: Proceedings of SIGMOD Conference 2011, pp. 1283-1286 (2008)

3. Kondylakis, H., Flouris, G., Plexousakis, D.: Ontology and Schema Evolution in Data Integration: Review and Assessment. In: Meersman, R., Dillon, T., Herrero, P. (eds.) OTM 2009, Part II. LNCS, vol. 5871, pp. 932-947. Springer, Heidelberg (2009)

4. Ferrara, A., Lorusso, D., Montanelli, S., Varese, G.: Towards a Benchmark for Instance Matching. In OM (2008)

5. Fellegi, I., Sunter, A.: A theory for record linkage. Journal of the American Statistical Association 64(328), 1183-1210 (1969)

6. Newcombe, H.B., Kennedy, J.M., Axford, S.J., James, A.P.: Automatic Linkage of Vital Records. Science 130(3381), 954-959 (1959)

7. Elmagarmid, A.K., Ipeirotis, P.G., Verykios, V.S.: Duplicate Record Detection: A Survey. IEEE Trans. Knowl. Data Eng. 19(1), 1-16 (2007)

8. Bhattacharya, I., Getoor, L.: Entity resolution in graphs. In: Mining Graph Data. Wiley \& Sons (2006)

9. Hernandez, M.A., Stolfo, S.J.: The merge/purge problem for large databases. SIGMOD Rec. 24(2), 127-138 (1995)

10. Noessner, J., Niepert, M., Meilicke, C., Stuckenschmidt, H.: Leveraging Terminological Structure for Object Reconciliation. In: Aroyo, L., Antoniou, G., Hyvönen, E., ten Teije, A., Stuckenschmidt, H., Cabral, L., Tudorache, T. (eds.) ESWC 2010, Part II. LNCS, vol. 6089, pp. 334-348. Springer, Heidelberg (2010)

11. Meghini, C., Doerr, M., Spyratos, N.: Managing Co-reference Knowledge for Data Integration. In: EJC 2008, pp. 224-244 (2008)

12. Kondylakis, H., Doerr, M., Plexousakis, D.: Mapping Language for Information Integration. Technical Report 385, ICS-FORTH, pp: 1-10 (2006)

13. Kondylakis, H., Doerr, M., Plexousakis, D.: Empowering Provenance in Data Integration. In: Grundspenkis, J., Morzy, T., Vossen, G. (eds.) ADBIS 2009. LNCS, vol. 5739, pp. 270-285. Springer, Heidelberg (2009)

14. Euzenat, J., Shvaiko, P.: Ontology Matching. Springer, Heidelberg (2007)

15. Wang, C., Lu, J., Zhang, G.: Integration of Ontology Data through Learning Instance Matching. In: Web Intelligence 2006, pp. 536-539 (2006)

16. Isaac, A., van der Meij, L., Schlobach, S., Wang, S.: An Empirical Study of InstanceBased Ontology Matching. In: Aberer, K., Choi, K.-S., Noy, N., Allemang, D., Lee, K.-I., Nixon, L.J.B., Golbeck, J., Mika, P., Maynard, D., Mizoguchi, R., Schreiber, G., CudréMauroux, P. (eds.) ASWC 2007 and ISWC 2007. LNCS, vol. 4825, pp. 253-266. Springer, Heidelberg (2007) 
17. Schopman, B.A.C., Wang, S., Schlobach, S.: Deriving Concept Mappings through Instance Mappings. In: Domingue, J., Anutariya, C. (eds.) ASWC 2008. LNCS, vol. 5367, pp. 122-136. Springer, Heidelberg (2008)

18. Castano, S., Ferrara, A., Lorusso, D., Montanelli, S.: On the Ontology Instance Matching Problem. In: DEXA Workshops 2008, pp. 180-184. IEEE Computer Society (2008)

19. Tang, J., Li, J., Liang, B., Huang, X., Li, Y., Wang, K.: Using Bayesian Decision for Ontology Alignment. J. Web Semantics 4(4), 243-262 (2006)

20. Jean-Mary, Y.R., Kabuka, M.R.: ASMOV: Ontology Alignment with Semantic Validation. In: Joint SWDB-ODBIS Workshop, Vienna, Austria, pp. 15-20 (September 2007)

21. Stoermer, H., Rassadko, N., Vaidya, N.: Feature-Based Entity Matching: The FBEM Model, Implementation, Evaluation. In: Pernici, B. (ed.) CAiSE 2010. LNCS, vol. 6051, pp. 180-193. Springer, Heidelberg (2010)

22. Nagy, M., Vargas-Vera, M., Stolarski, P.: DSSim Results for OAEI. In: OM (2009)

23. Hanif, M.S., Aono, M.: MPEG-7 Based Multimedia Information Integration through Instance Matching. In: ICSC, pp. 618-623 (2009)

24. Engmann, D., Maßmann, S.: Instance Matching with COMA++. In: BTW Workshops, pp. 28-37 (2007)

25. Huber, J., Sztyler, T., Noessner, J., Meilicke, C.: CODI: Combinatorial Optimization for Data Integration - Results for OAEI 2011. In: OM (2011)

26. Castano, S., Ferrara, A., Lorusso, D., Montanelli, S.: The HMatch 2.0 Suite for Ontology Matchmaking. In SWAP (2007)

27. Hu, W., Chen, J., Cheng, G., Qu, Y.: ObjectCoref \& Falcon-AO: Results for OAEI 2010. In: OM (2010)

28. Araujo, S., Hidders, J., Schwabe, D., de Vries, A.P.: SERIMI - Resource Description Similarity, RDF Instance Matching and Interlinking. CoRR, Vol. abs/1107.1104 (2011).

29. Cruz, I.F., Antonelli, F.P., Stroe, C.: Efficient Selection of Mappings and Automatic Quality-driven Combination of Matching Methods. In OM (2009)

30. Niu, X., Rong, S., Zhang, Y., Wang, H.: Zhishi.links Results for OAEI 2011. In: OM (2011)

31. Sa1s, F., Niraula, N., Pernelle, N., Rousset, M.-C.: LN2R-A knowledge based reference reconciliation system: OAEI 2010 Results. In: OM (2010)

32. Jena Semantic Web Framework for Java, http: / / jena. sourceforge.net/

33. Kalaitzaki, M.: Design and implementation of a system for semantic schema matching. Master Thesis - University of Crete, Department of Computer Science (November 2009)

34. Manakanatas, D.: Design and Implementation of a tool for semi-automated semantic schema matching. Master Thesis - University of Crete, Department of Computer Science (April 2006)

35. Doerr, M., Papagelis, M.: A Method for Estimating the Precision of Placename Matching. IEEE Trans. Knowl. Data Eng, 1089-1101 (2007)

36. The ISLab Instance Matching Benchmark, http: / islab.dico.unimi . it/iimb/

37. Svatek, V., Svab-Zamazal, O.: Empirical knowledge discovery over ontology matching results. In: Proc. 1st ESWC International Workshop on Inductive Reasoning and Machine Learning on the Semantic Web, Heraklion, Greece (2009)

38. Euzenat, J., Ferrara, A., Hollink, L., Isaac, A., Joslyn, C., Malaisé, V., Meilicke, C., Nikolov, A., Pane, J., Sabou, M., Scharffe, F., Shvaiko, P., Spiliopoulos, V., Stuckenschmidt, H., Sváb-Zamazal, O., Svátek, V., Santos, C.T.D., Vouros, G.A., Wang, S.: Results of the Ontology Alignment Evaluation Initiative 2009. In: OM (2009)

39. Hanif, M.S., Aono, M.: Anchor-Flood: Results for OAEI 2009. In: OM (2009)

40. Jean-Mary, Y.R., Shironoshita, M., Kabuka, M.R.: ASMOV: Results for OAEI 2009. In: OM (2009) 
41. Nagy, M., Vargas-Vera, M., Stolarski, P.: DSSim Results for OAEI. In: OM (2009)

42. Stoermer, H., Rassadko, N.: Results of OKKAM Feature based Entity Matching Algorithm for Instance Matching Contest of OAEI, In: OM (2009)

43. Zhang, X., Zhong, Q., Shi, F., Li, J., Tang, J.: RiMOM Results for OAEI, In: OM (2009)

44. Flouris, G., Manakanatas, D., Kondylakis, H., Plexousakis, D., Antoniou, G.: Ontology Change: Classification and Survey. Knowledge Engineering Review (KER) 23(2), 117-152 (2008)

45. Euzenat, J., Ferrara, A., Hollink, L., Isaac, A., Joslyn, C., Malaisé, V., Meilicke, C., Nikolov, A., Pane, J., Sabou, M., Scharffe, F., Shvaiko, P., Spiliopoulos, V., Stuckenschmidt, H., Sváb-Zamazal, O., Svátek, V., Santos, C.T.D., Vouros, G.A., Wang, S.: Results of the Ontology Alignment Evaluation Initiative. In: OM (2009) 